\title{
Conocimiento y uso de método anticonceptivo de emergencia en estudiantes universitarios, Pereira, Colombia
}

\author{
Awareness and use of emergency contraception methods among university students in Pereira, Colombia
}

\author{
Germán Oved Acevedo-Osorio1* orcid.org/0000-0002-1499-2579 \\ Nicolás Ramírez-García' orcid.org/0000-0001-5248-1561 \\ Julián David Cardona-Osorio' orcid.org/0000-0003-1240-7409 \\ Christian Alexander Oliveros-Ruiz' orcid.org/0000-0003-0622-9642
}

1 Grupo de Investigación Los Asépticos-Fundación Universitaria del Área Andina - Seccional Pereira, Colombia

\section{Resumen}

Introducción: Los métodos anticonceptivos de emergencia o también llamados contracepción poscoital, es una de las alternativas que pueden ser utilizadas para evitar un embarazo no deseado en mujeres que han tenido relaciones sexuales sin protección, hasta 72 horas después de la relación. Objetivo: Establecer el nivel de conocimiento y el uso de los métodos anticonceptivos de emergencia utilizados en estudiantes universitarios. Materiales y métodos: Estudio observacional, descriptivo y transversal. Se incluyeron estudiantes de 15 a 25 años de una Institución de Educación Superior en el Municipio de Pereira, Colombia con vida sexual activa. Con un total de 127 estudiantes donde se incluyeron variables sociodemográficas de conocimiento y uso de métodos. Resultados: Se encuestaron 127 estudiantes donde la mediana en la edad fue de 19 años en sexo femenino con un rango intercuartílico de 3 y el sexo masculino presento una mediana de 21 años con un rango intercuartílico de 5 . El condón fue el método más utilizado por los encuestados con un 35,4\%, seguido de las píldoras con un 26,8\%. Conclusiones: El método de elección fue el condón y existe alta prevalencia de consumo de métodos anticonceptivos de emergencia, con desconocimiento frente a su uso.

Palabras clave: Anticonceptivos; anticonceptivos poscoito; conocimiento. (Fuente: DeCS, Bireme).

\begin{abstract}
Introduction: The emergency contraceptive method, also known as post-coital contraception, is one of the alternatives that can be used to avoid an unwanted pregnancy up to 72 hours after a woman has had unprotected sexual intercourse. Objective: To establish the level of awareness and practice of emergency contraceptive methods among university students. Materials and methods: An observational, descriptive and cross-sectional study. The sample included 15- to 25-year-old students of a Higher Education Institution from the Municipality of Pereira- Colombia, with an active sexual life. The study included sociodemographic variables with respect to awareness and practice of contraceptive methods. Results: 127 students were surveyed, where the median age for female students was 19 years (IQR of 3), whereas male students showed a median age of 21 years old (IQR of 5). The condom was the most commonly used contraceptive method, used by $35.4 \%$ of the students, followed by contraceptive pills, which was used by $26.8 \%$ of students. Conclusions: The birth control method of choice was condom and although there is a high prevalence of consumption of emergency contraceptive methods, students show little awareness of their use.
\end{abstract}

Key words: Contraceptive agents; contraceptives, poscoito; knowledge. (Source: DeCS, Bireme). 


\section{Introducción}

Los anticonceptivos de emergencia (AE), son un método simple, seguro y efectivo para la planificación familiar que pueden utilizar las mujeres para prevenir un embarazo no planeado(1). Los AE se usan días después de un relación sexual no protegida, es decir, cuando no se usó un anticonceptivo de barrera, cuando este falló o fue usado incorrectamente y en el caso extremo de una violación sexual; este disminuye el porcentaje de jóvenes en embarazo y de abortos, permitiendo ejercer a las mujeres el derecho a controlar su fertilidad(2).

Desde la década de los 70's, se desarrolló un mecanismo que evitaba el embarazo después de la relación sexual a través del denominado método Yuzpe, posteriores investigaciones hicieron que este método fuera reemplazado por la administración de levonorgestrel por cuanto no presentaba los efectos adversos del Yuzpe y tenía mayor efectividad(3). Existen varios métodos $\mathrm{AE}$ y cada uno de ellos es efectivo si se usa dentro de un rango de tiempo específico, así el método Yuzpe debe administrarse antes de las 72 horas, el consumo de progestinas o de acetato de ulipristal debe realizarse dentro de las 120 horas posteriores al coito, al igual que el uso del Dispositivo Intrauterino (DIU); después de este tiempo su efectividad disminuye ${ }^{(4)}$.

China lidera el uso de $\mathrm{AE}$ con un $44 \%$, seguido de Suecia 40\%, Suiza 20\%, México 16\% y Estados Unidos $14 \%$ y en regiones de Brasil oscila entre el 13\% y el $32 \%$. Esto se ve reflejado en el apoyo del país a los jóvenes y en su asequibilidad; sin embargo, también influyen aspectos individuales como no vivir en un núcleo familiar, condiciones socioeconómicas y otros factores relacionados a su entorno social(5).

Según el Ministerio de Salud, en Colombia el 99\% de los abortos se realizan de manera clandestina, estimando así, unos 400.000 abortos cada año, por esta razón es considerado como un problema de salud pública; solo 3.400 fueron legales para el año 2015 . En la ciudad de Bogotá la tasa de abortos fue de 66 por cada 1000 mujeres y en la región oriental de 18 por cada 1000 mujeres(6). Según la Organización Mundial de la Salud (OMS) y el Instituto Guttmacher de 2010 a 2014 se realizaron 25 millones de abortos peligrosos en todo el mundo ( $45 \%$ del total); la mayoría de abortos peligrosos (97\%) se produjo en países en desarrollo de África, Asia y América Latina. En
Latinoamérica solo 1 de cada 4 abortos se realizó de manera segura, estos representan un bajo riesgo de mortalidad para la madre gestante(7).

Según la encuesta nacional de demografía y salud de 2010 podemos analizar un aumento del embarazo adolescente: 1 de cada 5 adolescentes colombianas de 15 a 19 años ha estado en embarazo en algún momento, $16 \%$ ya son madres y $4 \%$ están esperando su primer hijo, actualmente las cifras son demasiado altas y siguen siendo de interés social y político(8).

Según OMS, cuando una mujer no utiliza métodos anticonceptivos y tiene relaciones durante la etapa fértil de su ciclo menstrual, existe un $8 \%$ de probabilidad de quedar en embarazo; disminuyendo a $1 \%$ si consume progestágeno y al $2 \%$ si utiliza $\mathrm{AE}$ que contiene estrógenos ${ }^{(9)}$. Por lo anterior, el objetivo de este estudio es establecer el nivel de conocimiento y el uso de los métodos anticonceptivos de emergencia utilizados en estudiantes universitarios, de una institución de educación superior en el municipio de Pereira, Colombia, con el fin de orientar políticas en salud pública desde el nivel local, especialmente en el ámbito educativo universitario.

\section{Materiales y métodos}

Estudio observacional, descriptivo y transversal. Se incluyeron adolescentes de 18 a 25 años con vida sexual activa, estudiantes de una institución de educación superior en el municipio de Pereira, Colombia.

\section{Población y muestra}

La población estuvo conformada por 2.300 estudiantes universitarios mayores de 18 años, de la cual se obtuvo una muestra de 364 estudiantes. Los criterios de inclusión fueron: estudiantes matriculados legal y financieramente en el periodo del año estudiado, mayores de 18 años y aceptación voluntaria de participación en el estudio. Se consideró criterio de exclusión, el no haber tenido relaciones sexuales, puesto que este es un factor primordial para el cumplimiento del objetivo del estudio. Con base en estos criterios la muestra final fue de 127 estudiantes.

\section{Recolección de la información}

Para la recolección de la información se utilizó un cuestionario de conocimientos, actitudes y prácticas de los estudiantes universitarios frente al consumo de métodos de planificación de emergencia. Validado 
por expertos en el área de psicología de bienestar universitario, consta de 19 preguntas sobre datos sociodemográficos de los participantes y variables de estudio del conocimiento acerca de métodos anticonceptivos, anticonceptivos de emergencia, su uso y aplicación.

\section{Análisis de la información}

La información fue recolectada mediante Google Forms y tabulada en Excel $2015^{\circledR}$ y analizadas con el software estadístico SPSS versión 23. Para las variables cualitativas se realizó un análisis de frecuencias y para las cuantitativas se utilizaron medidas de tendencia central y dispersión. Se realizó un análisis bivariado, para la relación entre conocimiento y uso de métodos anticonceptivos de emergencia con prueba Chi-cuadrado y un valor de significancia de $\mathrm{p}<0,05$.

\section{Consideraciones éticas}

Esta investigación fue aprobada por el Comité de Investigación de la Facultad de Salud de la Fundación Universitaria del Área Andina y considerada como un estudio sin riesgo según la Resolución № 8430 de 1993 del Ministerio de Salud de Colombia, que establece las normas científicas, técnicas y administrativas para la investigación en salud. La participación fue voluntaria y posterior a la firma de consentimiento informado se realizó el diligenciamiento de la encuesta.

\section{Resultados}

Se analizó la información de 127 participantes, de estos el $73,2 \%$ correspondieron al sexo femenino; la edad presentó con una mediana de 20 años y un rango intercuartílico de 4; no se encontró diferencias significativas entre la edad y el haber consumido píldoras anticonceptivas de emergencia $(p=0,633)$. La población de sexo masculino presentó una mediana de 21 años con un rango intercuartílico de 5, las mujeres presentaron una mediana de 19 años con un rango intercuartílico de 3. Se encontró diferencias significativas $(p=0,013)$, entre las medianas de las edades entre hombres y mujeres.

El condón es el método anticonceptivo más utilizado por los encuestados con un $35,4 \%$, seguido de las píldoras con un 26,8\%. El consumo de métodos anticonceptivos de describe en la Tabla 1. Al momento indagar sobre el conocimiento de los $\mathrm{AE}, \mathrm{El}$ $76,4 \%$ de las personas encuestadas respondieron que se debe utilizar antes de las 24 horas después del coito, seguido del 7,1\% que respondieron que debía utilizarse antes de las 72 horas. El 30,2\% había utilizado el AE luego de consumir alcohol y realizar prácticas sexuales sin protección.

Tabla 1. Caracterización de uso de métodos anticonceptivos

\begin{tabular}{|c|c|c|c|}
\hline & & $\mathbf{n}$ & $\%$ \\
\hline \multirow[t]{2}{*}{ Sexo } & Femenino & 93 & 73,2 \\
\hline & Masculino & 34 & 26,8 \\
\hline \multirow[t]{2}{*}{ Relaciones sexuales } & $\mathrm{Si}$ & 111 & 87,4 \\
\hline & No & 16 & 12,6 \\
\hline \multirow[t]{2}{*}{ ¿Usted o su pareja ha estado embarazada? } & $\mathrm{Si}$ & 5 & 3,9 \\
\hline & No & 122 & 96,1 \\
\hline \multirow[t]{2}{*}{ Uso métodos anticonceptivos } & $\mathrm{Si}$ & 92 & 72,4 \\
\hline & No & 35 & 27,6 \\
\hline \multirow[t]{6}{*}{ Métodos anticonceptivos } & Condón & 45 & 35,4 \\
\hline & Píldoras & 34 & 26,8 \\
\hline & Inyectables & 24 & 18,9 \\
\hline & DIU & 7 & 5,5 \\
\hline & Método del ritmo & 4 & 3,1 \\
\hline & Coito interrumpido & 2 & 1,6 \\
\hline
\end{tabular}

La frecuencia de uso de $\mathrm{AE}$ entre 1 y 2 veces en el último año es de 52,3\%, evidenciando que más de la mitad de los encuestados ha utilizado este método, la causa más frecuente para usar esta alternativa fue el no uso de método anticonceptivo durante la relación sexual $(19,7 \%)$, seguido de la ruptura o fallo del condón $(17,3 \%)$. Por otra parte, el $63,8 \%$ recibió apoyo de la pareja para hacer uso de AE y en un 59\% de los casos fue un amigo(a) quien recomendó el uso de AE (Tabla 2). 
Tabla 2. Descripción del uso de métodos anticonceptivos de emergencia

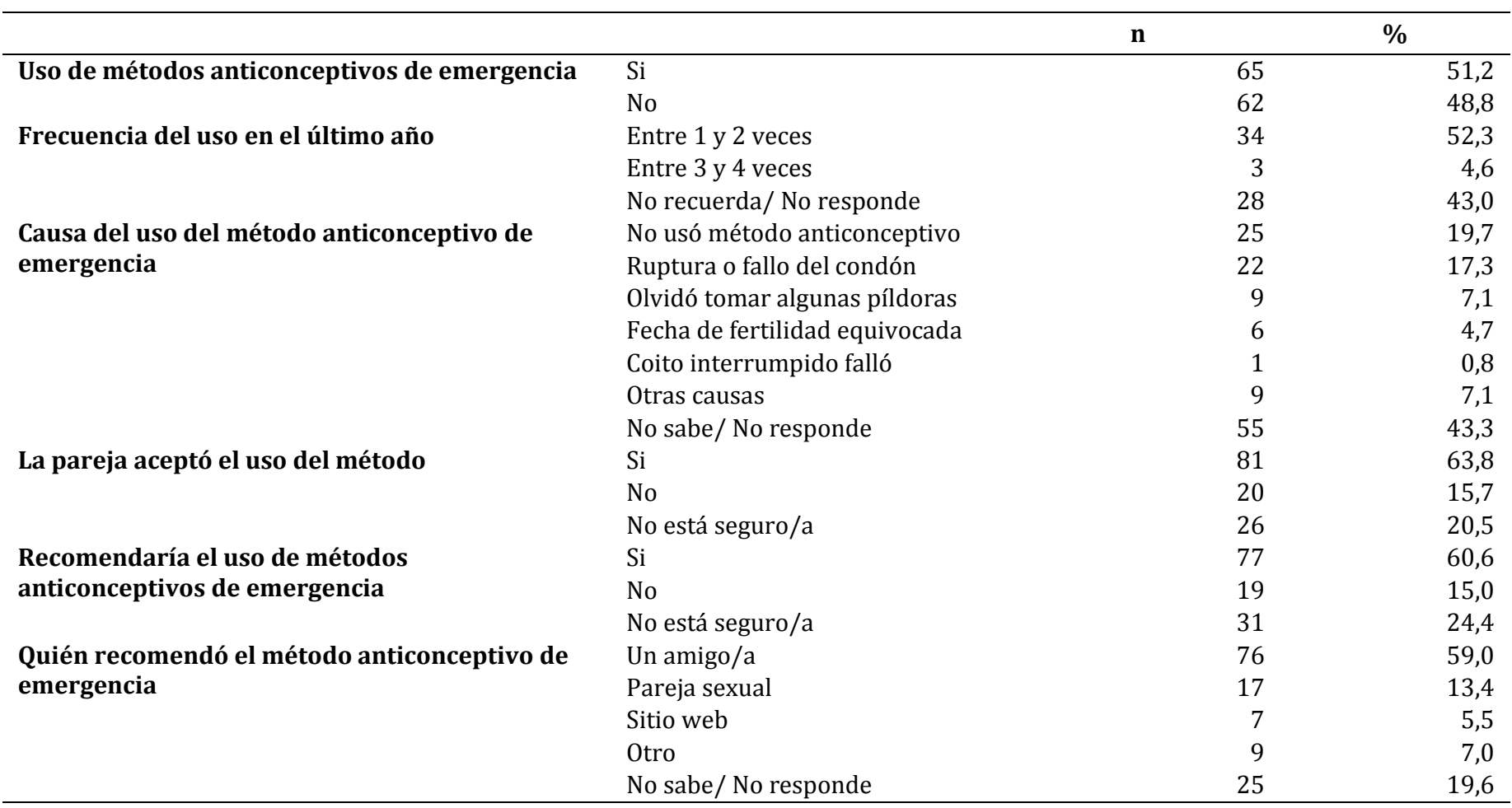

Al realizar el análisis por género, se pudo evidenciar que el $26,5 \%$ de las parejas de los hombres entrevistados utilizaron el método $\mathrm{AE}$ debido a que no utilizaron ningún método anticonceptivo, seguido por el $20,6 \%$ que lo usaron por falla del condón. En el grupo de las mujeres entrevistadas el 17,2\% lo utilizaron por no haber usado ningún método anticonceptivo, 16,1\% por ruptura o fallo del condón, $8,6 \%$ por olvidar tomar la píldora y el 5,4\% debido al mal uso del calendario del ritmo. Se encontraron diferencias significativas entre el género y las causas para haber usado el método $\mathrm{AE}(\mathrm{p}=0,002)$ (Tabla 3$)$.

Tabla 3. Relación entre el sexo y consumo de métodos anticonceptivos

\begin{tabular}{|c|c|c|c|c|}
\hline & & & Sexo & \\
\hline & & $\begin{array}{c}\text { Femenino } \\
\mathrm{N}(\%)\end{array}$ & $\begin{array}{c}\text { Masculino } \\
\text { N (\%) }\end{array}$ & Valor P \\
\hline Anticonceptivo de & $\mathrm{Si}$ & $43(46,2)$ & $22(64,7)$ & 0,065 \\
\hline emergencia & No & $50(53,8)$ & $12(35,3)$ & \\
\hline Causa del uso del método & No usó método anticonceptivo & $16(17,2)$ & $9(26,5)$ & 0,002 \\
\hline anticonceptivo de & Ruptura o fallo del condón & $15(16,1)$ & $7(20,6)$ & \\
\hline emergencia & Olvidó tomar algunas píldoras & $8(8,6)$ & $1(1,6)$ & \\
\hline & Fecha de fertilidad equivocada & $5(5,4)$ & $1(2,9)$ & \\
\hline & Coito interrumpido falló & $0(0,0)$ & $1(2,9)$ & \\
\hline & Otras causas & $2(2,2)$ & $7(20,6)$ & \\
\hline La pareja aceptó el uso del & $\mathrm{Si}$ & $56(60,2)$ & $25(73,5)$ & 0,057 \\
\hline método & No & $19(20,4)$ & $1(2,9)$ & \\
\hline & No está seguro & $18(19,4)$ & $8(23,5)$ & \\
\hline
\end{tabular}

\section{Discusión}

Se pudo evidenciar que un gran porcentaje de estudiantes utilizan anticoncepción de emergencia de una manera inadecuada (45\%) y además desconocen la forma de utilización, este desconocimiento del adecuado uso, impide beneficiarse del método anticonceptivo para su correcta función, asociado a un consumo desmedido de $\mathrm{AE}$ relacionado a su fácil adquisición, como se plantea en el estudio de Sánchez, 
et al.(1), el uso de estos métodos anticonceptivos se asocia al consumo excesivo de alcohol y a relaciones sexuales de forma recreativa, cabe esto resaltar que el desconocimiento también se asocia al no trabajar temas de sexualidad en las aulas en su escolaridad y al no tratarlos en sus hogares, esto conlleva a una maternidad y paternidad prematura que conducen a estos jóvenes a una precaria vida adulta a la cual no están preparados, además de poner la vida en riesgo de la madre gestante y él bebe como lo plantean Sam, et al.(10), y Chofakian, et al.(5).

Dado que los AE son métodos utilizados para evitar un embarazo no planeado, es importante tener en cuenta que cada uno debe usarse dentro de un plazo estipulado para que sus efectos sean los deseados(5). Estos son considerados métodos de planificación legales, sin embargo es importante resaltar que una adecuada educación sexual en los adolescentes, es de gran importancia para evitar embarazos prematuros(11).

Un estudio realizado por Lapeira, et al., acerca de conocimientos, creencias y prácticas en anticoncepción de los adolescentes de la cultura Caribe, encontró que estos iniciaron tenían relaciones a temprana edad con más de un compañero sexual, un $35 \%$ tenían relaciones sexuales sin protección, revelando que el hombre tiene una mayor influencia sobre la mujer al momento de utilizar métodos anticonceptivos y que tres de cada cinco menores de 25 años aseguraron que les falta más educación sexual en los ámbitos educativos y familiares ${ }^{(12) .}$

En un estudio realizado por Sili-Francisco, et al., en 2018 se pudo evidenciar que el $45 \%$ de los estudiantes encuestados de un total 244, manifestaron tener bajo conocimiento sobre estos métodos de planificación y los utilizaban de manera incorrecta, donde el 44,4\% de las mujeres tenía un bajo nivel de conocimiento(13), similar a lo encontrado en el presente estudio donde las mujeres tuvieron un mayor conocimiento frente al uso de los AE que los hombres. Por su parte, Tapia, et al., plantea que las mujeres continúan siendo las responsables culturalmente de la planificación, existiendo una doble moral frente a la sexualidad, por cuanto existe mayor permisividad para los hombres, coincidiendo con este estudio donde el 20,4\% del consumo de AE no fue consentido por la pareja(14).
Con respecto a los conocimientos y prácticas de anticoncepción se encontró que Moreno, et al., en el año 2015 obtuvo 148 encuestas, de las cuales el 9\% de los estudiantes de enfermería y el 35,7\% de los estudiantes de medicina, tienen conocimiento sobre el método de anticoncepción de emergencia(15); en nuestro el presente estudio se evidenció que existe un alto consumo de $\mathrm{AE}$ relacionado a prácticas sexuales riesgosas e inadecuadas y el 52,3\% de los estudiantes encuestados lo han utilizado.

Algunas jóvenes que utilizan de manera inadecuada los AE no tienen conocimiento previo y asertivo de cuáles son las posibles complicaciones y riesgos, por ello es importante implementar programas de prevención sobre embarazo no deseado y anticoncepción de emergencia, donde el uso de algunos medios informativos serían de gran ayuda para convalidar la importancia del buen manejo de los métodos de anticoncepción de emergencia, por cuanto como se plantea en algunos estudios, existe una amplia disponibilidad y eficacia, pero como intervención de salud pública resulta una experiencia "decepcionante"(16). Cárdenas-García, et al., en un estudio sobre el uso de la anticoncepción de emergencia en un grupo de 107 estudiantes universitarias, el 76,6\% usaron el condón(17). Gogna, et al., en su estudio de anticoncepción y maternidad en el año 2017 encontraron que los métodos más utilizados fueron la píldora $(40,7 \%)$ y el preservativo $(37,0 \%)$ y de menor uso el inyectable $(8,2 \%)^{(18)}$, resultado similar al de este estudio donde el preservativo tuvo un 35,4\% de uso entre la población encuestada.

Se observó que para el uso de los métodos $\mathrm{AE}$ el $63,8 \%$ recibió apoyo de su pareja, algo similar a lo reportado por Covarrubias, et al., en su estudio sobre la utilización de métodos anticonceptivos en estudiantes de enfermería en el año 2016, donde el $56 \%$ de los hombres manifestaron haber comprado la píldora "del día después" para su pareja, y de ellos el $34 \%$ había recurrido a ella en más de una ocasión en el último año(19).

Es necesario que previamente al uso de algún método anticonceptivo se reciba la información clara y veraz por parte de personal capacitado, a fin de prevenir embarazos no deseados y reducir la tasa de embarazos tempranos; al respecto el estudio de Guerrero, et al., con estudiantes de la Facultad de Medicina, encontró que un $60 \%$ de los encuestados 
refirió que la primera fuente a la cual acudiría en busca de información sobre métodos anticonceptivos, sería el personal de salud(20); resultados similares se obtuvieron en la investigación realizada en mujeres que utilizaron la píldora anticonceptiva de emergencia en Honduras, el 38\% de ellas recibió información en los centros de salud o en el hospital y $21 \%$ recibió instrucción en los centros educativos ${ }^{(21)}$. Sin embargo, en este estudio se evidenció que el 59\% recibió orientación del uso de AE por un amigo(a), coincidiendo con el estudio de Mandujano, et al., donde el $81 \%$ de las jóvenes encuestadas utilizaron $A E$, debido a la recomendación del farmacéutico o de un amigo(a) (22).

\section{Conclusión}

Existe un alto consumo de anticonceptivos de emergencia atribuible entre otras cosas al desconocimiento de sus indicaciones, riesgos $y$ contraindicaciones; por ello se sigue considerando un método de anticoncepción de rutina, aumentando el riesgo de embarazo no deseado. De esta manera se convierte en una prioridad, la educación sexual en estudiantes universitarios, por cuanto carecen de un adecuado nivel de conocimiento sobre anticoncepción de emergencia.

\section{Recomendaciones}

La población universitaria participante cuenta con características sociodemográficas y de consumo similar a las reportadas por la literatura, sin embargo, la selección aleatoria de población y los criterios de inclusión redujo significativamente la muestra, hecho que podría representar una limitación del estudio. Para mejorar este aspecto se recomienda en un futuro realizar estudios discriminados por género o incluir únicamente el género femenino.

\section{Conflicto de intereses}

No se declaran conflictos de interés con relación a esta investigación.

\section{Referencias}

1. Sánchez-Meneses MC, Dávila-Mendoza R, Ponce-Rosas ER. Conocimiento y uso de métodos anticonceptivos en adolescentes de un centro de salud. Aten Fam [Internet]. 2015;22(2):35-8. Disponible en: https://www.medigraphic.com/pdfs/atefam/af2015/af152b.pdf

2. Leyva-López A, Chávez-Ayala R, Atienzo EE, Allen-Leigh B, Ramírez-Villalobos D, Yunes-Díaz E, et al. Anticoncepción de emergencia en estudiantes mexicanos. Salud Pública Mex [Internet]. 2010;52(2):156-64. Disponible en: https://www.scielosp.org/article/ssm/content/raw/?resou rce_ssm_path=/media/assets/spm/v52n2/v52n2a08.pdf

3. Morán-Faúndes JM. La anticoncepción de emergencia en Chile: estructuración de su demanda en función de variables socioeconómicas. Rev Bras Estud Popul [Internet]. 2013;30(1):125-44. Disponible en: http://www.scielo.br/pdf/rbepop/v30n1/v30n1a07

4. Ministerio de Salud y Protección Social. Anticoncepción de emergencia [Internet]. Bogotá: MinSalud; 2017. Disponible en:

https://www.minsalud.gov.co/salud/publica/ssr/Paginas/ Anticoncepcion-de-emergencia.aspx

5. Chofakian CBN, Vilela-Borges AL, Sayuri-Sato AP, PereiraAlencar G, Alves- dos Santos O, Fujimori E. Does the knowledge of emergency contraception affect its use among high school adolescents?. Cad Saude Publica [Internet]. 2016;32(1):1-11. Disponible en: http://www.scielosp.org/scielo.php?script=sci_arttext\&pid =S0102-311X2016000100703

6. Ministerio de Salud y Protección Social. Interrupción voluntaria del embarazo, un derecho humano de las mujeres [Internet]. Bogotá: MinSalud; 2016. Disponible en: https://www.minsalud.gov.co/sites/rid/Lists/BibliotecaDig ital/RIDE/VS/PP/abc-maternidad-elegida.pdf

7. Organización Mundial de la Salud. En todo el mundo se producen aproximadamente 25 millones de abortos peligrosos al año. Ginebra: OMS; 2017.

8. Morón-Duarte LS, Latorre C, Tovar JR. Risk factors for adolescent pregnancy in Bogotá, Colombia, 2010: a casecontrol study. Rev Panam Salud Pública [Internet]. 2014 [citado el 15 de abril de 2019];36:179-84. Disponible en: https://www.scielosp.org/pdf/rpsp/2014.v36n3/179184/en\#

9. Organización Mundial de la Salud. Planificación familiar: Un manual mundial para proveedores [Internet]. Ginebra: OMS; $2011 . \quad$ Disponible en: http://apps.who.int/iris/bitstream/10665/44074/1/9780 978856304_spa.pdf

10. Sam-Soto S, Osorio-Caballero M, Rodríguez-Guerrero RE, Pérez-Ramírez NP. Comportamiento sexual y anticoncepción en la adolescencia. Acta pediátrica México [Internet]. 2014;35(2):159-65. Disponible en: http://www.scielo.org.mx/scielo.php?script=sci_arttext\&pi $\mathrm{d}=\mathrm{S} 0186-23912014000600008$

11. Asensio A, Nebot L, Estruga L, Perez G, Diez È. Contraception in the Roma population living in two low-income neighborhoods of Barcelona (Spain). Gac Sanit [Internet]. 2019;33(2):119-26. Disponible en: https://doi.org/10.1016/j.gaceta.2017.11.011

12. Lapeira-Panneflex P, Acosta-Salazar D, Vasquez-Munive M. Conocimientos, creencias y prácticas de los adolescentes de la cultura caribe en anticoncepción. Rev Cuid [Internet]. 2016;7(1):10-5. Disponible en: http://www.scielo.org.co/scielo.php?script=sci_arttext\&pid =S2216-09732016000100008\&lng=en\&nrm=iso\&tlng=es

13. Sili-Francisco PS, Pelaez-Mendoza J. Nivel de conocimientos sobre la anticoncepción hormonal de emergencia en estudiantes de Medicina. Rev Cuba Obstet y Ginecol [Internet]. 2018;44(2):0. Disponible en: http://www.revginecobstetricia.sld.cu/index.php/gin/articl e/view/343

14. Tapia-Martínez H, González-Hernando C, Puebla-Nicolás E. 
Influencia de determinantes de género en la anticoncepción de estudiantes de Enfermería y Obstetricia mexicanas. Enfermería Univ [Internet]. 2017;14(4):251-8. Disponible en: http://dx.doi.org/10.1016/j.reu.2017.08.002

15. Moreno CL, Sepúlveda LE. Conocimientos y prácticas en anticoncepción de los estudiantes de medicina y enfermería de Manizales, Colombia 2015. Rev Chil Obstet Ginecol. 2017;82(3):259-64.

16. Crosignani PG. Emergency contraception. Widely available and effective but disappointing as a public health intervention: A review. Hum Reprod. 2015;30(4):751-60.

17. Cárdenas-García LJ, Sánchez-Zamora ME, Ramírez-de la Roche OF, Robledo-Domínguez A. Uso de la anticoncepción de emergencia en un grupo de estudiantes universitarias. Atención Fam [Internet]. 2018;21(3):90-3. Disponible en: http://dx.doi.org/10.1016/S1405-8871(16)30025-6

18. Gogna M, Binstock G. Anticoncepción y maternidad: Hallazgos de un estudio cuanti-cualitativo con adolescentes de 18 y 19 años de cuatro provincias argentinas. Salud Colect. 2017;13(1):63-72.

19. Covarrubias E, Ramírez Villegas R, Ramírez Villegas R, Verde
Flota EE de J, Verde Flota EE de J, Rivas Espinosa JG, et al. Utilización de Métodos Anticonceptivos en estudiantes de Enfermería. Investig en Enfermería Imagen y Desarro. 2015;18(1):31.

20. Guerrero-Ortiz HA, Benavides-Espinoza M, GuzmanBarrantes EK, Meneses-Prieto CC. Nivel de conocimientos, actitudes y practicas sobre métodos anticonceptivos en estudiantes de medina humana "Daniel Alcides Carrion" Ica 2017. Rev Médica Panacea [Internet]. 2017;6(3):116-22. Disponible en: http://revpanacea.unica.edu.pe/index.php/RMP/article/vie $\mathrm{w} / 202$

21. Alas C, Calix K, Velázquez F, Bejarano S. Perfil de mujeres que utilizan la píldora anticonceptiva de emergencia en El Progreso, Yoro, Honduras (2014-2015). Rev Hispanoam Ciencias la Salud. 2016;2(2):132-9.

22. Mandujano-Contreras JC, Reyes-de la Cruz C, SarmientoArena Z, Murillo-Palma AL, Osorio-Alejo DR. Conocimiento y uso de la píldora de emergencia en jóvenes universitarias de Tabasco. Horiz Sanit. 2018;17(3):227-34. 